\title{
BIOMIMICRY AND ITS APPLICATIONS -
}

\section{A REVIEW}

\author{
Mayank Chaurasia \\ Department of Life Sciences and Biotechnology \\ Graphic Era University, Dehradun, Uttarakhand, India \\ Soumya Srivastava \\ Department of Life Sciences and Biotechnology \\ Graphic Era University, Dehradun, Uttarakhand, India
}

\begin{abstract}
Biomimicry is the study of imitating nature and its components in order to decipher the problems of design for human society in a sustainable way. This Biomimetics aims at imitating in non-living systems the features of a living biological system; this is separate from replicating or testing the biological system itself. Natural selection is the main process over geological time for the evolution of living organisms with a well-adapted structure and material. Nature has solved all of our issues, some of the most interesting help is immunity to environmental damage, self-healing, self-assembly, and artificial skin and antibacterial properties.
\end{abstract}

Keywords- Biomimicry, Gecko lizard (Gekkota), Multifocal optical system, Nephila spp., Spider dragline silk

\section{INTRODUCTION}

Human beings are the most progressive species on Earth, but for the coming generations he has created enormous, continuous problems. Luckily, we're equipped with answers to our own problems.

Biomimicry is an access to innovation through the strategies of mimic nature which seeks sustainable solutions towards human challenges. The goal is to create systems and goods that will change the ways of living and standardize life on earth for a longer period.

I think at the intersection of Biology and Technology would be the most enticing and successful inventions for this $21 \mathrm{st}$ century. A modern definition of "the fittest survival".

Increasing powerful wind power, generating sustainable development, reducing energy usage, increasing high grain yields, improving soil and water quality for future generation, innovative pain free injections with easy penetration in surgery, responsive pressure sensors for tsunami detection before reaching shore, artificial skin made of spider silk, lotus-inspired self-cleaning substance, more powerful cameras and multifocal contact lenses influenced by Gecko lizard (Gekkota) with multifocal optical system, 350 times better sensitive than human beings, Artificial cochlear radio chip allowing portable devices effective of running radio, telephone calls and internet services, Bat's SONAR (Sound Navigation And Ranging) system designed to make navigation sticks for blind people identifying the position of obstacles, Robotic arms such as elephant trunk support disabled people as elephant trunk has more power due to 40,000 muscles and Cicada(Cicadidae) wings have antibacterial property, light antireflection, superhydrophobic surface and unique tribology-manufactured $\mathrm{Si}$ wet-process nanostructure shows antibacterial property to E. Coli[1].

Owing to planar structures, hydraulic mechanisms, increasing stiffness, elastic mechanisms, contractile mechanisms, and aerodynamic structure and processes such as $3 \mathrm{D}$ printing, polyacrylamide gel and soft and hard piezo ceramic materials[2], conversion of human ear electrical and mechanical signals, the above items are possible only.

Within this review, I concentrate within greater depth on two of its application. Now, is it artificial skin made from spider silk? The human skin cells put on these meshes (silk mesh) flourished when the proper nutrients, warmth and air were provided. This is a sandwich-like arrangement with two primary types of skin cells, keratinocytes and fibroblasts, the tissue-like form resembles epidermis, the outermost skin layer, and dermis, the layer of living tissue below the epidermis that includes capillaries of blood, nerve endings, sweat glands, hair follicles, and other structures[3].

Gecko Lizard called 'Gecko Vision' is inspired by the second script. Gecko lizards are nocturnal, and one of the species that can able to see colours at night, and the discovery by scientists of a set of distinct focused zones will lead to insight into better cameras and lenses[4]. The multifocal optical system consists of large cones which, at the human colour vision level, the researchers measured that they are 350 times more sensitive than human cone vision. We are interested in designing more powerful cameras and multifocal contact lenses inspired by this little guy with eyes 350 times more sensitive than humans.

\section{SPIDER SILK MADE ARTIFICIAL SKIN}

In the field of plastic reconstructive surgery, the development of new ground-breaking matrices for skin repair is in urgent need. Ideal biomaterial would encourage cell attachment, 


\section{International Journal of Engineering Applied Sciences and Technology, 2020 \\ Vol. 4, Issue 12, ISSN No. 2455-2143, Pages 545-549 \\ Published Online April 2020 in IJEAST (http://www.ijeast.com)}

growth and proliferation. It can also degrade without releasing harmful substances but does not exert a defective immune response over a reasonable period. Nephila spp. spider dragline silk largely satisfies these demands.

Skin grafting is important for treating burn victims. The total cost of burn-repair surgery in India is approx. Rs2,27,000.

Rather than using skin from a human, scientists concentrate more on the artificial substance that works more stably on the skin. Ideally, a graft will be of a body-tolerated material and have its own skin cells inserted in it to replace missing tissue, and the most important thing is that it would regenerate with time as the new skin grows in and would be powerful enough to endure all the limbs of ordinary skin encounters. The strongest biomaterial known is Spider silks. Besides this there is a lot of possible medical benefit of webs, such as combating infections, stopping bleeding, repairing wounds and acting as artificial ligaments. Spider silk's exceptional strength and stretchiness characteristics provide quick and simple handling and transfer of many forms of implants.

The key importance of this spider silk is that it does not induce any immune response or body rejection reactions.

Power and durability are generally called material property and is mutually exclusive. The science and technology of polymeric fibre, the quest for a very solid and hard fiber continues. Exploring the limit of strength and resilience of fibrous materials and, most particularly, the durability of silk with human living skin cells, is of practical and scientific importance.

Nephila Spp. Spider dragline web may be used for the production of skin cells as a biological matrix. One significant feature of tissue engineering is the study of the biologically decaying biocompatible material[5]. Varity of silk are produced from different silk gland for web development, which includes cocoons, orb webs, or dragline silk. Dragline thread, a protective line for the spider, from Nephila spp[6]. The spiders have been thoroughly studied. It consists of 5 layers (from the outside to the inside): a protective lipid shell, a water balance glycocoat, a skin layer and the outer and inner core comprised of major ampullate proteins. Most institutes and industries utilize silk biomaterials as practicable for biomaterial application but neither of them used native spider dragline silk as a matrix for the cultivation of skin cells. Spider and silkworm silks vary in several different characteristics: the immunogenic serinic coat of bombyx mori silk contributes to an immune response and must be extracted but even bombyx mori silk is well tolerated in vivo and still commonly used as biomaterial[7]. Like Spider silk, native spider does not induce such an in vivo immunogenic response and can be used without further processing. In order to obtain silk from
Nephila spp., spiders must be reared in large rooms, where they must be fed with crickets and tended intensively with regard to their web building environments proportional to their body size. Today it is not possible to produce significant amounts of spider silk (for industrial standards). Bombyx mori silk rearing is less time- and space-consuming but many factors must be considered, such as the temperature of rearing. Therefore, a lot of work is underway to develop large quantities of recombinant silk spider proteins. Several techniques have been developing transgenic silkworm, producing spider silk proteins, combining the chemical and physical properties of spider silk with facilitated bombyx mori silk harvesting. Alternative approaches included spider silk protein processing in plants, insect cells or bacteria. All of these methods have certain disadvantages, whether proteins are generated in cells or bacteria, they must first be processed and then spun into fibers in complex procedures, but for all that, the biomechanical properties of many newly created artificial fibers vary from natural spider silk fibres.

\subsection{Methodology}

The native spider dragline silk was woven on steel frames, harvested directly from Nephila spp. spiders. Constructions were sterilized and fibro blasted to seed. Keratinocytes were added to produce a bilayered skin model, consisting of dermis and epidermis equivalents after two weeks of cultivation of single fibroblasts. Constructions in co-culture were lifted over an originally constructed setup for the production of air / liquid interfaces for the next three weeks. Constructions were embedded in paraffin after the cultivation phase with a specially designed spider silk system to prevent super contraction. Parts of paraffin cross-sections were stained for microscopic examination in Haematoxylin \& Eosin $(\mathrm{H} \& \mathrm{E})[8]$.

\subsection{A Breeding of spiders}

Nephila spp. requires separate breeding space, and temperature $>17^{\circ} \mathrm{C}$ and 70 percent humidity should be maintained. Both male and female spiders were held together; spiders cocoons were collected and put in glass cases to hatch and reach a suitable size[9], [10].

\subsection{B Harvesting of spider dragline silk}

For the collection of silk, only adult female spiders were used by placing the spiders backwards on a polystyrene cube with a gauze bandage and needles. The dragline silk could be separated from the spinneret by stimulating the Main ampullate gland and spun onto a coil or frame[11]. Spiders were fed with Acheta domesticus and water after collection of the silk[12].

\subsection{Frame design, weaving and culturing}




\section{International Journal of Engineering Applied Sciences and Technology, 2020 \\ Vol. 4, Issue 12, ISSN No. 2455-2143, Pages 545-549 \\ Published Online April 2020 in IJEAST (http://www.ijeast.com)}

Weaving frames were made, straight wires of stainless steel with a diameter of $0.7 \mathrm{~mm}$ were bent to small weaving frames with side lengths from $1-1.5 \mathrm{~cm}$. Frames were put into a special, self-made tuning tool for the selection of spider silk. In cross patterns, spider silk was woven onto the frame to achieve a mesh size of 10-100 $\mu \mathrm{m}$. In this way, two meshes were generated: one mesh at the top and one at the bottom side of the frame at the distance of the wire's diameter $(0.7$ $\mathrm{mm})$. The $0.7 \mathrm{~mm}$ interspaced between the upper and lower mesh of the frame was placed into a spider silk clew. Before usage, frames were steam-sterilized at $121^{\circ} \mathrm{C}$ with a pressure of 2 bars and $100 \%$ moisture. In vivo, skin cells build the interface of the body and therefore have contact from one side to the ambient air as well as nutrients from the other side must be supplied[13].

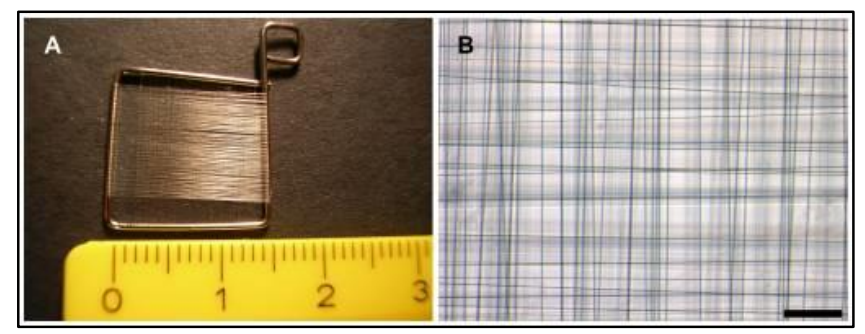

Figure 1: Frame Design

The constructs must be cultivated at the air liquid interface to generate these physiological conditions which enhance the organotypical cell growth. Thus, frames were raised on a silicone scale packed with polymer fibres. The medium surface was below frame level. Both were linked by the polymer fibres, that enabled the supply of nutrients and the evacuation of metabolites by diffusion.

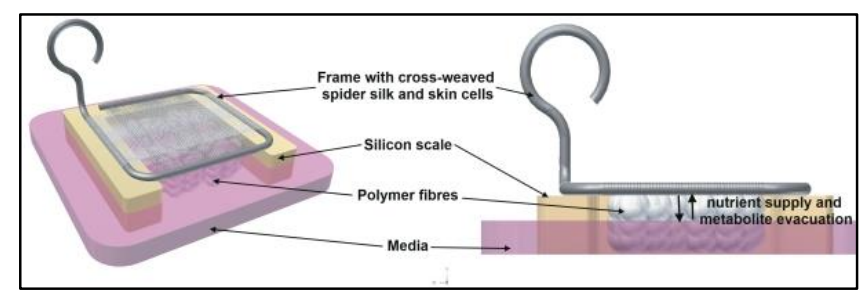

Figure 2: Air Liquid interface for cultivation

\subsection{Cell culturing}

Human keratinocytes (Human Adult Calcium High Temperature Keratinocytes, $\mathrm{HaCaT}$ ) have been grown in $\mathrm{HaCaT}$-specific medium to avoid cell adhesion to the bottom of cell culture dishes. Mouse embryonic fibroblasts (MEF) have been grown in MEF-specific medium containing Dulbecco's modified Eagle medium (DMEM) high glucose cell medium.

The 3D skin model Frames with cross-woven spider silk were mounted in a well and a $100 \mu \mathrm{l}$ cell suspension with $1 \times 106$
MEF in a MEF-specific medium was mounted in the center of the frame. Placed in an incubator for 30 minutes to facilitate cell attachment on the silk fiber plates, later filled with $2 \mathrm{ml} \mathrm{MEF-specific} \mathrm{medium} \mathrm{and} \mathrm{cultivated} \mathrm{for} \mathrm{two}$ weeks. Three times a week, the media had changed.

\subsection{E Immunohistology and histology}

After cultivation, the time frames were set with $4 \%$ buffered formalin, dehydrated in a graded sequence of-alcohol concentrations, cleared in xylene, enclosed in the standard paraffin procedure and sliced into $10 \mu \mathrm{m}$ sections, and slides deparaffinated, rehydrated by descending alcohol concentrations, stained with haematoxyline and eosine (H\&E) or submitted for immunohistological ana. Cell viability assay was carried out in order to assess the amount of living cells in the seeded frames.

\section{MULTIFOCAL CONTACT LENSES INSPIRED BY GECKO LIZARD}

Tarentola chazaliae, the nocturnal helmet gecko, actively works in dim moonlight when humans are color blind. At the color vision threshold, the sensitivity of the helmet gecko eye was measured to be 350 times greater than that of human cone vision. The gecko's optics and large cones are significant reasons for using color vision at low luminous intensities[13]. Use photorefractometry and a high-resolution adapted Hartmann - Shack wavefront camera, we also demonstrate that the helmet gecko's optical system has distinct localized zones of different refractive powers, a so-called multifocal optical system. The intraspecific variability is great, but the zones varied by 15 diopters in most individuals studied. This is of the same magnitude as required to concentrate light on the wavelength range to which the most sensitive gecko photoreceptors are at. We equate the helmet gecko's optical system to that of the Phelsuma madagascariensis grandis, a diurnal day gecko. The day gecko's optical system shows no signs of distinct concentrated zones and thus is monofocal[14].

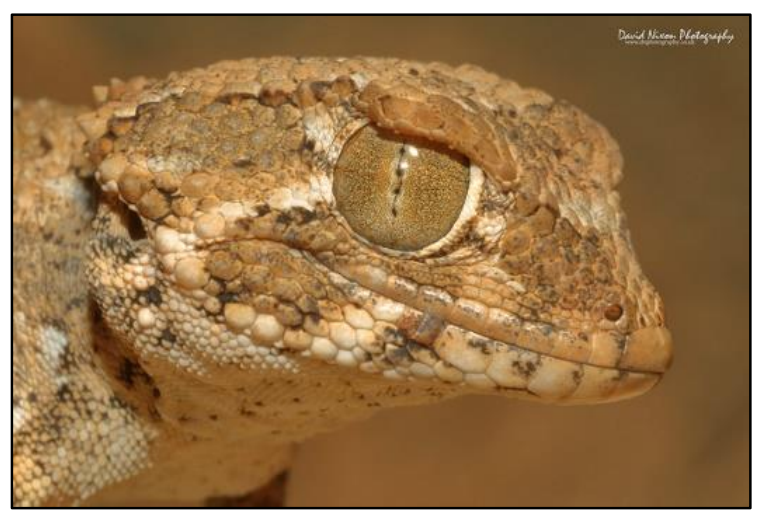

Figure 3: Noctural Gecko (Tarentola chazaliae) eyes 


\section{International Journal of Engineering Applied Sciences and Technology, 2020 \\ Vol. 4, Issue 12, ISSN No. 2455-2143, Pages 545-549 \\ Published Online April 2020 in IJEAST (http://www.ijeast.com)}

Diurnal lizards evolved and their eyes lost all rods and cones of the standard vertebrate duplex retina and left only with various types of single and double cones. Around some stage in evolution, however, a group of lizards switched the Geckos to a noturnal lifestyle. The cones of nocturnal geckos have become much larger and more light-sensitive than those of their diurnal relatives, in response to the demands of nocturnal vision without rods[15]. Nocturnal Geckos has maintained their three separate UV, Green and Blue sensitive photo pigments and their eyes are sensitive enough to get color information at night[16]. The nocturnal helmet geckos, Tarentola chazaliae, could distinguish colors in a dual choice of behaviour, at intensities corresponding to dim moonlight. Its eyes are round and entirely extended at these dim light intensities. Throughout this report, we investigated the pupil, eye dimensions, and cone dimensions of helmet geckos and used those data to measure optical sensitivity in order to understand the adaptations that enable the animals to see colors under dim light conditions. In particular, longitudinal chromatic aberration affects eyes designed for night vision, such as the eyes of nocturnal geckos with a wide pupil and a short focal length, $\mathrm{f}$. As a result, the light of short wavelengths is more intensely refracted and thus oriented closer to the lens than the long wavelengths. If this is not fixed for nocturnal vision, the retinal picture may be significantly distorted in an eye adjusted to it. It has been proposed that multifocal optical systems with distinct localized zones of different refractive powers correct some of the defocus on the retina caused by chromatic aberration[17]. Kroger et al. have shown the nocturnal gecko's eyes have multifocal optical system. We would be able to build more efficient cameras and maybe even useful multifocal contact lenses with the information from the gecko eyes.

\section{BIOMIMICRY A SOURCE OF INSPIRATION}

Individual designers are influenced in many ways by nature and solutions.

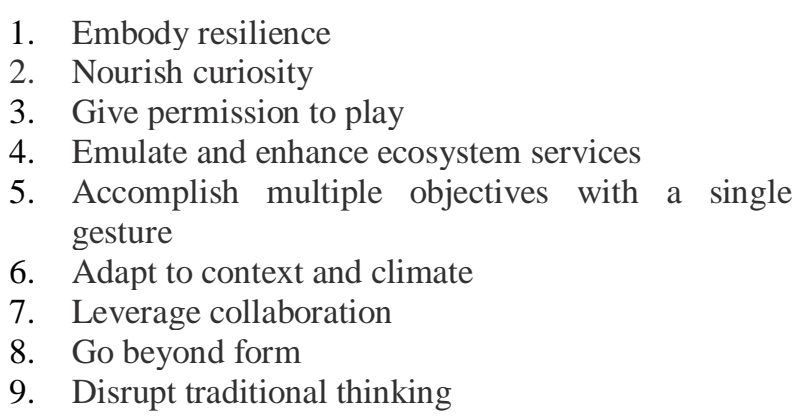

\section{CONCLUSION}

Native spider dragline silk woven on steel frames offers an ideal matrix for 3-dimensional skin cell cultivation. Both the fibroblast and keratinocyte cell lines bind to and proliferate the silk spider fibres. Driven by the spider silk fibers, they sprout into the clusters, and assemble in about a week. A wellbalanced, bilayered cocultivation can be accomplished by serum elimination, change of medium conditions and cultivation time at the air / liquid interphase in two continuously segregated strata. So, spider silk appears to be a promising biomaterial for enhancing skin regeneration. Multi-focal optical system of Nocturnal gecko eyes from which we may build more advance and highly effective cameras and hopefully usable multi-focal contact lenses with knowledge of gecko eyes. Biomimicry presents itself as a basis, a foundation of a new methodology of research rather than mere serendipity. Biomimicry needs to be approached in a multidisciplinary way to consider the laws of nature in order to arrive at a comprehensive design solution.

\section{ACKNOWLEDGEMENT}

Foremost, we would like to express our sincere gratitude to Dr. (Ms.) Jigisha Anand, Assistant Professor, Dept. of Biotechnology, Graphic Era (Deemed to be University). Last but not the least, we would like to thank our family for support.

\section{REFERENCE}

[1] X. Zhang, L. Wang, and E. Levänen, "Superhydrophobic surfaces for the reduction of bacterial adhesion," $R S C A d v$., vol. 3, no. 30, pp. 12003-12020, 2013, doi: 10.1039/c3ra40497h.

[2] S. Shahab, S. Zhao, and A. Erturk, "Soft and Hard Piezoelectric Ceramics and Single Crystals for Random Vibration Energy Harvesting," Energy Technol., vol. 6, no. 5, pp. 935-942, 2018, doi: 10.1002/ente.201700873.

[3] D. [Updated 2018 D. 6]. I. S. [Internet]. T. I. (FL): S. P. 2020 J.-. A. from: https://www. ncbi. nlm. nih. gov/books/NBK535346. Brown TM, Krishnamurthy K. Histology, "Histology, Dermis."

[4] L. Roth and O. Biology, "' Gecko vision ': Key to the multifocal contact lens of the future?," no. May, pp. 1-2, 2009.

H. Wendt et al., "Artificial Skin - Culturing of different skin cell lines for generating an artificial skin substitute on Cross-Weaved spider silk fibres," PLoS One, vol. 6, no. 7, 2011, doi: 10.1371/journal.pone.0021833.

[6] S. Salehi, K. Koeck, and T. Scheibel, "Spider silk for tissue engineering applications," Molecules, vol. 


\section{International Journal of Engineering Applied Sciences and Technology, 2020 \\ Vol. 4, Issue 12, ISSN No. 2455-2143, Pages 545-549 \\ Published Online April 2020 in IJEAST (http://www.ijeast.com)}

25, no. 3, 2020, doi: 10.3390/molecules 25030737 .

[7] Charu Vepari; David L. Kaplan, "Silk as

Biomaterial," Prog. Polym. Sci., vol. 100, no. 2, pp. 130-134, 2007, doi:

10.1016/j.pestbp.2011.02.012.Investigations.

[8] V. Hauptmann, N. Weichert, M. Rakhimova, and U. Conrad, "Spider silks from plants - A challenge to create native-sized spidroins," Biotechnol. J., vol. 8, no. 10 , pp. $1183-1192,2013$, doi: 10.1002/biot.201300204.

[9] A. L. Cory and J. M. Schneider, "Effects of social information on life history and mating tactics of males in the orb-web spider Argiope bruennichi," Ecol. Evol., vol. 8, no. 1, pp. 344-355, 2018, doi: 10.1002/ece3.3672.

[10] S. E. Jones, "Influence of Temperature and Humidity on the Life History of the Spider Agelena Naevia Walckenaer1," Ann. Entomol. Soc. Am., vol. 34, no. 3, pp. 557-571, 1941, doi: 10.1093/aesa/34.3.557.

[11] R. W. Work and P. D. Emerson, "An apparatus and technique for the forcible silking of spiders," $J$. Arachnol., vol. 10, no. 1, pp. 1-10, 1982, doi: $10.2307 / 3705113$.

[12] L. Römer and T. Scheibel, "The elaborate structure of spider silk: structure and function of a natural high performance fiber.," Prion, vol. 2, no. 4, pp. 154-161, 2008, doi: 10.4161/pri.2.4.7490.

[13] A. Steins et al., "In vitro evaluation of spider silk meshes as a potential biomaterial for bladder reconstruction," PLoS One, vol. 10, no. 12, 2015, doi: 10.1371/journal.pone.0145240.

[14] L. S. V. Roth, L. Lundström, A. Kelber, R. H. H. Kröger, and P. Unsbo, "The pupils and optical systems of gecko eyes," J. Vis., vol. 9, no. 3, pp. 111, 2009, doi: 10.1167/9.3.27.

[15] B. Röll, "Gecko vision-visual cells, evolution, and ecological constraints," J. Neurocytol., vol. 29, no. 7, pp. 471-484, 2000, doi: 10.1023/A:1007293511912.

[16] L. S. V. Roth and A. Kelber, "Nocturnal colour vision in geckos," Proc. R. Soc. B Biol. Sci., vol. 271, no. SUPPL. 6, pp. 485-487, 2004, doi: 10.1098/rsbl.2004.0227.

[17] R. H. H. Kroger, M. C. W. Campbell, R. D. Fernald, and H. J. Wagner, "Multifocal lenses compensate for chromatic defocus in vertebrate eyes," J. Comp. Physiol. A Sensory, Neural, Behav. Physiol., vol. 184, no. 4, pp. 361-369, 1999, doi: $10.1007 / \mathrm{s} 003590050335$. 